Fatiha Alabau-Boussouira (Metz)

\title{
ASYMPTOTIC STABILITY OF WAVE EQUATIONS WITH MEMORY AND FRICTIONAL BOUNDARY DAMPINGS
}

Abstract. This work is concerned with stabilization of a wave equation by a linear boundary term combining frictional and memory damping on part of the boundary. We prove that the energy decays to zero exponentially if the kernel decays exponentially at infinity. We consider a slightly different boundary condition than the one used by M. Aassila et al. [Calc. Var. 15, 2002]. This allows us to avoid the assumption that the part of the boundary where the feedback is active is strictly star-shaped. The result is based on multiplier techniques and integral inequalities.

1. Introduction. We are interested in energy decay of a wave equation subjected to a boundary damping combining frictional and memory effects. The results presented here can be extended to more general nonlinear damping effects, as in [4], [8]. We refer more generally to [9], [16, 17], [18], [19], $[10],[6,7]$ for memory damping, to [11], [12] for polynomial type frictional damping (see also [14]), and to [13], [15] and [2, 3] for general nonlinear frictional damping.

We consider the following wave equation with frictional and memory boundary damping:

$$
\begin{aligned}
u_{t t}-\Delta u=0 & \text { on }(0,+\infty) \times \Omega, \\
u=0 & \text { on }(0,+\infty) \times \Gamma_{1}, \\
\frac{\partial u}{\partial \nu}=m \cdot \nu\left(-u_{t}+h * u\right) & \text { on }(0,+\infty) \times \Gamma_{0}, \\
u(0, \cdot)=u^{0}(\cdot), \quad u_{t}(0, \cdot)=u^{1}(\cdot) & \text { on } \Omega .
\end{aligned}
$$

2000 Mathematics Subject Classification: 35B35, 35B37, 45K05, 93D15, 93D20.

Key words and phrases: wave equation, stabilization, boundary damping, memory damping, integro-differential PDE's. 
Here $*$ stands for convolution, that is,

$$
(h * u)(t)=\int_{0}^{t} h(t-s) u(s) d s, \quad t \geq 0 .
$$

One can remark that the boundary condition differs from the one considered in [1]. In particular, the damping term includes an additional factor $m \cdot \nu$. This permits avoiding a condition like

$$
m \cdot \nu \geq \delta>0 \quad \text { on } \Gamma_{0} .
$$

The results presented below can be extended to nonlinear dampings. However, we prefer to keep the paper easy to read.

We assume that $\Omega$ is a non-empty bounded open set in $\mathbb{R}^{N}$ having a boundary $\Gamma$ of class $C^{2}$. Moreover, $\left\{\Gamma_{0}, \Gamma_{1}\right\}$ is a partition of $\Gamma$ such that $\bar{\Gamma}_{0} \cap \bar{\Gamma}_{1}=\emptyset$ and $x_{0}$ is a point in $\mathbb{R}^{N}$ such that $m \cdot \nu \geq 0$ on $\Gamma_{0}$ and $m \cdot \nu \leq 0$ on $\Gamma_{1}$, where $m(x)=x-x_{0}$. We set $R=\sup _{x \in \bar{\Omega}}|m(x)|$.

We set $H=L^{2}(\Omega)$ equipped with the usual norm, denoted by $|u|_{H}$, and $V=\left\{v \in H_{0}^{1}(\Omega): v=0\right.$ on $\left.\Gamma_{1}\right\}$, equipped with the norm $|\nabla u|_{H}^{2}$, equivalent to the usual Sobolev norm, thanks to Poincaré's inequality.

We denote by $\lambda_{0}^{2}>0$ the smallest constant such that

$$
\int_{\Gamma_{0}}|v|^{2} d \sigma \leq \lambda_{0}^{2} \int_{\Omega}|\nabla v|^{2} d x \quad \forall v \in V .
$$

Such a constant exists since the trace operator is continuous from $V$ to $L^{2}\left(\Gamma_{0}\right)$, and the $H^{1}$ seminorm is a norm on $V$.

The convolution kernel $h$ is assumed to be defined and differentiable from $[0, \infty)$ on $(0, \infty)$, and to satisfy

$$
h^{\prime}(t) \leq-\alpha h(t) \quad \forall t \geq 0,
$$

where $\alpha>0$ is a given constant, and

$$
\lambda_{0}^{2} R \int_{0}^{\infty} h(t) d t<1 .
$$

The results presented here can be extended to polynomially decaying kernels following the ideas of [4]. In this case, we prove that the energy decays polynomially with the same rate of decay as the kernel $h$ at infinity. These results can be obtained using nonlinear integral inequalities, and multipliers involving also appropriate powers of the energy.

We will use the following notation:

$$
(h \square u)(t)=\int_{0}^{t} h(t-s)\left(\int_{\Gamma_{0}} m \cdot \nu|u(t)-u(s)|^{2} d \sigma\right) d s .
$$


The paper is organized as follows. In Section 2, we recall well-posedness results, establish some preliminary results concerning for instance dissipation of the energy and give our main result. In Section 3, we prove exponential decay of the energy using appropriate multipliers and linear integral inequalities. Finally, in Section 4, we comment briefly on extensions of our results to other types of decay of the kernel, and state several open questions connected with this work.

2. Well-posedness and main result. We refer the reader to [1] for wellposedness in $\mathcal{C}([0, \infty) ; V) \cap \mathcal{C}^{1}([0, \infty) ; H)$ for initial data $\left(u^{0}, u^{1}\right) \in V \times H$. In this case, the solution is in a weak sense. If the initial data are more regular, that is, if $\left(u^{0}, u^{1}\right) \in\left(V \cap H^{2}(\Omega)\right) \times V$ and satisfies the compatibility condition

$$
\frac{\partial u^{0}}{\partial \nu}+u^{1}=0 \quad \text { on } \Gamma_{0}
$$

then the solution is strong, that is, $u \in \mathcal{C}\left([0, \infty) ; V \cap H^{2}(\Omega)\right) \cap \mathcal{C}^{1}([0, \infty) ; V) \cap$ $\mathcal{C}^{1}([0, \infty) ; H)$.

We define the energy of a solution as

$$
E(t)=\frac{1}{2}\left(\int_{\Omega}\left(\left|u_{t}\right|^{2}+|\nabla u|^{2}\right) d x-\int_{0}^{t} h(s) d s \int_{\Gamma_{0}} m \cdot \nu|u|^{2} d \sigma+h \square u\right) .
$$

Proposition 2.1. Let $u$ be a weak solution of (1.1), and $E$ its energy. Let $\eta>0$ be any number such that

$$
\eta<1-\lambda_{0}^{2} R \int_{0}^{\infty} h(s) d s
$$

Then

$$
E(t) \geq \frac{1}{2} \int_{\Omega} u_{t}^{2}+\eta \frac{1}{2} \int_{\Omega}|\nabla u|^{2} .
$$

Proof. Since $h \geq 0$, the definition of $E$ yields

$$
E(t) \geq \frac{1}{2} \int_{\Omega} u_{t}^{2}+\frac{1}{2} \int_{\Omega}|\nabla u|^{2}-\frac{1}{2} R \int_{0}^{\infty} h(s) d s \int_{\Gamma_{0}}|u|^{2} .
$$

From the definition of $\lambda_{0}$, we easily derive the desired estimate.

Lemma 2.2. Assume that $u$ is a weak solution of (1.1), and $E$ its energy. Then E satisfies the following dissipation relation: 


$$
\begin{aligned}
E(T)-E(S)= & -\int_{S}^{T} \int_{\Gamma_{0}} m \cdot \nu\left|u_{t}\right|^{2} d \sigma d t+\frac{1}{2} \int_{S}^{T} h^{\prime} \square u \\
& -\frac{1}{2} \int_{S}^{T} h(t) \int_{\Gamma_{0}} m \cdot \nu|u|^{2} d \sigma d t \quad \forall 0 \leq S \leq T .
\end{aligned}
$$

Moreover, for a strong solution, we have

$$
E^{\prime}(t)=-\int_{\Gamma_{0}} m \cdot \nu\left|u_{t}\right|^{2} d \sigma+\frac{1}{2} h^{\prime} \square u-\frac{h(t)}{2} \int_{\Gamma_{0}} m \cdot \nu|u|^{2} d \sigma \leq 0 .
$$

Proof. Let $u$ be a strong solution of (1.1). By the definition of $h \square u$, we have

$$
\begin{aligned}
& \frac{d}{d t}(h \square u)(t)=\left(h^{\prime} \square u\right)(t)+\frac{d}{d t}\left(\int_{0}^{t} h(s) d s \int_{\Gamma_{0}} m \cdot \nu|u|^{2} d \sigma\right) \\
& -h(t) \int_{\Gamma_{0}} m \cdot \nu|u|^{2} d \sigma-2\left(\int_{0}^{t} h(t-s) \int_{\Gamma_{0}} m \cdot \nu u(s) d \sigma d s\right) u_{t}(t) .
\end{aligned}
$$

Differentiating the energy (2.1) with respect to $t$, integrating by parts and using the boundary conditions in (1.1), we obtain

$$
\begin{aligned}
E^{\prime}(t)= & \int_{\Omega}\left(u_{t t}-\Delta u\right) u_{t} d x+\int_{\Gamma_{0}} m \cdot \nu\left(-\left|u_{t}\right|^{2}+\left(\int_{0}^{t} h(t-s) u(s) d s\right) u_{t}(t)\right) d \sigma \\
& -\frac{1}{2} \frac{d}{d t}\left(\int_{0}^{t} h(s) d s \int_{\Gamma_{0}} m \cdot \nu|u|^{2}\right)+\frac{1}{2} \frac{d}{d t}(h \square u)(t) .
\end{aligned}
$$

Applying now (2.6) in the above relation, we obtain (2.5). Integration of this last relation between $S$ and $T$ gives (2.4), which can be extended to weak solutions by usual density arguments.

We can now state our main result:

THEOREM 2.3. Assume the above hypotheses on $h$ and on $\Gamma_{0}$ and $\Gamma_{1}$. Then there exists $\omega>0$ such that for every initial data $\left(u^{0}, u^{1}\right) \in V \times H$, the corresponding energy satisfies

$$
E(t) \leq C E(0) e^{-\omega t} \quad \forall t \geq 0,
$$

where $C$ is a generic constant which does not depend on the initial data.

3. Proof of the exponential decay of energy. We prove Theorem 2.3 as follows. Let $u$ be a strong solution of (1.1). We introduce the multiplier

$$
M u=m \cdot \nabla u+\frac{n-1}{2} u
$$


and rewrite the left hand side of the following identity using appropriate integrations by parts and the boundary conditions satisfied by $u$ in (1.1):

This gives

$$
\int_{S}^{T} \int_{\Omega}\left(u_{t t}-\Delta u\right) M u d x d t=0 .
$$

$$
\begin{aligned}
\frac{1}{2} \int_{S}^{T} \int_{\Omega}\left(\left|u_{t}\right|^{2}+|\nabla u|^{2}\right)= & -\left[\int_{\Omega} u_{t} M u\right]_{S}^{T}+\frac{1}{2} \int_{S}^{T} \int_{\Gamma_{1}} m \cdot \nu\left|\frac{\partial u}{\partial \nu}\right|^{2} \\
& +\int_{S}^{T} \int_{\Gamma_{0}} m \cdot \nu(H+K),
\end{aligned}
$$

where $H$ and $K$ are given by

$$
H=\frac{1}{2}\left(\left|u_{t}\right|^{2}-|\nabla u|^{2}\right), \quad K=\left(u_{t}-h * u\right) M u .
$$

We estimate $K$ as follows:

$$
\begin{aligned}
& \left|\int_{S}^{T} \int_{\Gamma_{0}} m \cdot \nu K d \sigma d t\right| \leq \int_{S}^{T} \int_{\Gamma_{0}} m \cdot \nu\left(\left|u_{t}\right|+\left|\int_{0}^{t} h(t-s)(u(s)-u(t)) d s\right|\right. \\
& \left.+\left(\int_{0}^{t} h(s) d s\right)|u|\right)(R|\nabla u|)+\int_{S}^{T} \int_{\Gamma_{0}} m \cdot \nu\left(\left|\int_{0}^{t} h(t-s)(u(s)-u(t)) d s\right|\right. \\
& \left.+\left(\int_{0}^{t} h(s) d s\right)|u|\right) \cdot \frac{n-1}{2}|u|+\frac{n-1}{4}\left|\left[\int_{\Gamma_{0}} m \cdot \nu|u|^{2}\right]_{S}^{T}\right| \\
& \leq \frac{\gamma R^{2}}{2} \int_{S}^{T} \int_{\Gamma_{0}} m \cdot \nu|\nabla u|^{2} \\
& +C\left(\frac{1}{\gamma}+1\right) \int_{S}^{T} \int_{\Gamma_{0}} m \cdot \nu\left(\left|u_{t}\right|^{2}+\left(\int_{0}^{t} h(s) d s\right)\left(\int_{0}^{t} h(t-s)|u(t)-u(s)|^{2} d s\right)\right. \\
& \left.+\left(\int_{0}^{t} h(s) d s\right)|u|^{2}\right)+C E(S) \quad \forall \gamma>0 .
\end{aligned}
$$

We choose $\gamma=R^{-2}$. This gives, together with the property $m \cdot \nu \geq 0$ on $\Gamma_{0}$ and the above estimate,

$$
\begin{aligned}
\int_{S}^{T} \int_{\Gamma_{0}} m \cdot \nu & (H+K) \\
& \leq C \int_{S}^{T} \int_{\Gamma_{0}}\left(h \square u+m \cdot \nu\left(\left|u_{t}\right|^{2}+\left(\int_{0}^{t} h(s) d s\right)|u|^{2}\right)\right)+C E(S) .
\end{aligned}
$$


Hence,

$$
\begin{aligned}
\frac{1}{2} \int_{S}^{T} \int_{\Omega}\left(\left|u_{t}\right|^{2}+|\nabla u|^{2}\right) \leq-\left[\int_{\Omega} u_{t} M u\right]_{S}^{T}+\frac{1}{2} \int_{S}^{T} \int_{\Gamma_{1}} m \cdot \nu\left|\frac{\partial u}{\partial \nu}\right|^{2} \\
+C \int_{S}^{T}\left(h \square u+\int_{\Gamma_{0}} m \cdot \nu\left(\left|u_{t}\right|^{2}+\left(\int_{0}^{t} h(s) d s\right)|u|^{2}\right)\right)+C E(S) .
\end{aligned}
$$

We estimate the consecutive terms on the right hand side of (3.1). We first easily prove that the first term satisfies

$$
\left|\left[\int_{\Omega} u_{t} M u\right]_{S}^{T}\right| \leq C E(S) \quad \forall 0 \leq S \leq T .
$$

By the sign condition $m \cdot \nu \leq 0$ on $\Gamma_{1}$, the second term on the RHS of (3.1) is nonpositive. From the dissipation relation (2.4), we have

$$
\int_{S}^{T} \int_{\Gamma_{0}} m \cdot \nu\left|u_{t}\right|^{2} \leq E(S) \quad \forall 0 \leq S \leq T .
$$

Moreover, since $h$ is assumed to decay exponentially we have

$$
\int_{S}^{T} h \square u \leq-\frac{1}{\alpha} \int_{S}^{T} h^{\prime} \square u .
$$

Using once again the dissipation relation (2.4) in this last estimate, we obtain

$$
\int_{S}^{T} h \square u \leq \frac{2}{\alpha} E(S) \quad \forall 0 \leq S \leq T .
$$

To estimate the last term on the right hand side of (3.1) we proceed as follows. For $t \geq 0$ fixed, we consider the elliptic problem

$$
\begin{aligned}
\Delta z=0 & \text { on } \Omega, \\
z=0 & \text { on } \Gamma_{1}, \\
\frac{\partial z}{\partial \nu}=m \cdot \nu\left(\int_{0}^{t} h(s) d s\right) z+m \cdot \nu(h * u)(t) & \text { on } \Gamma_{0} .
\end{aligned}
$$

This elliptic problem is well-posed as we shall now see. Its variational formulation is to find $z$ in $V$ such that

$$
a(z, \phi)=L(\phi) \quad \forall \phi \in V,
$$

where $a$ is the continuous bilinear form on $V$ defined by

$$
a(z, \phi)=\int_{\Omega} \nabla z \cdot \nabla \phi-\left(\int_{0}^{t} h(s) d s\right) \int_{\Gamma_{0}} m \cdot \nu z \phi,
$$


and $L$ is the continuous linear form on $V$ defined by

$$
L(\phi)=\int_{\Gamma_{0}} m \cdot \nu(h * u) \phi .
$$

From the conditions (1.3)-(1.4) on $h$, we can easily show that $a$ is coercive on $V$. More precisely,

$$
a(\phi, \phi) \geq \eta|\nabla \phi|_{H}^{2} \quad \forall \phi \in V .
$$

The Lax-Milgram lemma implies that the above elliptic problem has a unique solution in $V$. By regularity theory, this solution is in $H^{2}(\Omega)$. Moreover, choosing $\phi=z$ in the above variational formula, and using (3.3), we obtain

$$
\begin{aligned}
\eta \int_{\Omega}|\nabla z|^{2} \leq & \int_{\Gamma_{0}} m \cdot \nu \int_{0}^{t} h(t-s)(u(s)-u(t)) z(t) d s d \sigma \\
& +\int_{0}^{t} h(s) d s \int_{\Gamma_{0}} m \cdot \nu u(t) z(t) d \sigma .
\end{aligned}
$$

Using Cauchy-Schwarz's and Young's inequalities, we obtain, for every $\delta>0$,

$$
\begin{aligned}
\eta \int_{\Omega}|\nabla z(t)|^{2} \leq & \frac{C}{\delta} \int_{\Gamma_{0}} m \cdot \nu \int_{0}^{t} h(t-s)|u(t)-u(s)|^{2} d s+\delta\left(\int_{0}^{t} h(s) d s\right) \int_{\Gamma_{0}} m \cdot \nu|z|^{2} \\
& +\frac{C}{\delta} \int_{0}^{t} h(s) d s \int_{\Gamma_{0}} m \cdot \nu|u(t)|^{2}+\delta \int_{0}^{t} h(s) d s \int_{\Gamma_{0}} m \cdot \nu|z(t)|^{2} .
\end{aligned}
$$

Using (1.2) and choosing $\delta$ sufficiently small, we obtain

$$
\int_{\Omega}|\nabla z(t)|^{2} \leq C_{1}(h \square u)(t)+C_{2} \int_{0}^{t} h(s) d s \int_{\Gamma_{0}} m \cdot \nu|u(t)|^{2} .
$$

From this estimate, the definition of the energy, (1.2) and (2.1), we deduce that

$$
\int_{\Omega}|\nabla z(t)|^{2} \leq C E(t) \quad \forall t \geq 0,
$$

and by (1.2) once again, we also have

$$
\int_{\Gamma_{0}} m \cdot \nu|z(t)|^{2} \leq C_{1}(h \square u)(t)+C_{2} \int_{0}^{t} h(s) d s \int_{\Gamma_{0}} m \cdot \nu|u(t)|^{2} \quad \forall t \geq 0 .
$$


We now multiply both sides of (3.4) by $h(t)$ and integrate the resulting inequality between $S$ and $T$ to obtain

$$
\begin{aligned}
\int_{S}^{T} \int_{\Gamma_{0}} h(t) m \cdot \nu|z(t)|^{2} \leq & C_{1} \int_{S}^{T} h(t)(h \square u)(t) \\
& +C_{2} \int_{S}^{T} \int_{0}^{t} h(s) d s \int_{\Gamma_{0}} m \cdot \nu h(t)|u(t)|^{2} \quad \forall t \geq 0 .
\end{aligned}
$$

Using now (1.3) together with the dissipation relation (2.4), we obtain

$$
\int_{S}^{T} \int_{\Gamma_{0}} h(t) m \cdot \nu|z(t)|^{2} \leq C E(S) \quad \forall 0 \leq S \leq T .
$$

We now consider the identity

$$
\int_{S}^{T} \int_{\Omega}\left(u_{t t}-\Delta u\right) z=0 .
$$

Integrating by parts the left hand side of this identity and using the boundary conditions in (1.1) together with those in (3.2), we obtain

$$
\begin{aligned}
\int_{S}^{T} \int_{0}^{t} h(s) d s \int_{\Gamma_{0}} m \cdot \nu|u(t)|^{2} \\
=\int_{S}^{T} \int_{\Omega} u_{t} z_{t}+\int_{S}^{T} \int_{\Gamma_{0}} m \cdot \nu\left[-u_{t}(t) z(t)+\left(\int_{0}^{t} h(t-s)(u(t)-u(s)) d s\right) u(t)\right. \\
\left.\quad-\left(\int_{0}^{t} h(t-s)(u(t)-u(s)) d s\right) z(t)\right] d \sigma d t-\left[\int_{\Omega} u_{t} z\right]_{S}^{T} \cdot
\end{aligned}
$$

Hence, estimating the right hand side, we obtain, for each $\varepsilon>0$ and $\delta>0$,

$$
\begin{aligned}
\int_{S}^{T} \int_{0}^{t} h(s) d s \int_{\Gamma_{0}} m \cdot \nu|u(t)|^{2} \leq \delta \int_{S} \int_{\Omega}\left|u_{t}\right|^{2}+\frac{C}{\delta} \int_{S}^{T} \int_{\Omega}\left|z_{t}\right|^{2}+\frac{C}{\varepsilon} \int_{S}^{T} \int_{\Gamma_{0}} m \cdot \nu\left|u_{t}\right|^{2} \\
+\varepsilon \int_{S}^{T} \int_{\Gamma_{0}} m \cdot \nu|z|^{2}+\frac{C}{\varepsilon} \int_{S}^{T} h \square u+\varepsilon \int_{S}^{T} \int_{\Gamma_{0}} m \cdot \nu \int_{0}^{t} h(s) d s|u(t)|^{2} \\
+\varepsilon \int_{S}^{T} \int_{\Gamma_{0}} m \cdot \nu \int_{0}^{t} h(s) d s|z(t)|^{2}+C E(S) \quad \forall 0 \leq S \leq T .
\end{aligned}
$$


Using the above estimates on $z$, together with the inequality (1.3) on $h$ and the dissipation relation (2.4), we obtain

$$
\begin{aligned}
& \int_{S}^{T} \int_{0}^{t} h(s) d s \int_{\Gamma_{0}} m \cdot \nu|u(t)|^{2} \leq \delta \int_{S}^{T} \int_{\Omega}\left|u_{t}\right|^{2}+\frac{C}{\delta} \int_{S}^{T} \int_{\Omega}\left|z_{t}\right|^{2} \\
& +C\left(\frac{1}{\varepsilon}+1\right) E(S)+C \varepsilon \int_{S}^{T} \int_{\Gamma_{0}} m \cdot \nu \int_{0}^{t} h(s) d s|u(t)|^{2} \quad \forall 0 \leq S \leq T .
\end{aligned}
$$

Hence, choosing $\varepsilon$ sufficiently small, we have, for every $\delta>0$,

$$
\begin{aligned}
& \int_{S}^{T} \int_{0}^{t} h(s) d s \int_{\Gamma_{0}} m \cdot \nu|u(t)|^{2} \\
& \quad \leq \delta \int_{S}^{T} \int_{\Omega}\left|u_{t}\right|^{2}+\frac{C}{\delta} \int_{S}^{T} \int_{\Omega}\left|z_{t}\right|^{2}+C E(S) \quad \forall 0 \leq S \leq T .
\end{aligned}
$$

We now need an estimate on the $L^{2}$ norm of $z_{t}$ in $[S, T] \times \Omega$. For this, we differentiate the solution $z$ of the elliptic problem (3.2) with respect to time and take $z_{t}$ as a test function. This gives

$$
\begin{aligned}
\int_{\Omega}\left|\nabla z_{t}\right|^{2}-\left(\int_{0}^{t} h(s) d s\right) \int_{\Gamma_{0}} m \cdot \nu\left|z_{t}\right|^{2}=\int_{\Gamma_{0}} m \cdot \nu h(t) z z_{t} \\
\quad+\int_{\Gamma_{0}} m \cdot \nu \int_{0}^{t} h^{\prime}(t-s)(u(s)-u(t)) z_{t}(t) d s+\int_{\Gamma_{0}} m \cdot \nu h(t) u(t) z_{t}(t) .
\end{aligned}
$$

Hence, for all $\gamma>0$ we obtain

$$
\begin{aligned}
\eta \int_{S} \int_{\Omega}\left|\nabla z_{t}\right|^{2} \leq & \gamma \int_{S}^{T} \int_{\Gamma_{0}} m \cdot \nu h(t)\left|z_{t}\right|^{2}+\frac{C}{\gamma} \int_{S}^{T} \int_{\Gamma_{0}} m \cdot \nu h(t)|z|^{2} \\
& +\frac{C}{\gamma} \int_{S}^{T}\left(-h^{\prime} \square u\right)+\frac{C}{\gamma} \int_{S}^{T} \int_{\Gamma_{0}} m \cdot \nu h(t)|u(t)|^{2} \\
& +\gamma h(0) \int_{S}^{T} \int_{\Gamma_{0}} m \cdot \nu\left|z_{t}\right|^{2}+\gamma \int_{S}^{T} \int_{\Gamma_{0}} m \cdot \nu h(t)\left|z_{t}\right|^{2} .
\end{aligned}
$$

We now use (3.5) in the above estimate, together with (1.2) and once again the dissipation relation (2.4). If we choose $\gamma$ sufficiently small, this gives

$$
\int_{S}^{T} \int_{\Omega}\left|\nabla z_{t}\right|^{2} \leq C E(S) \quad \forall 0 \leq S \leq T .
$$


We use this last estimate in (3.6) to obtain

$$
\int_{S}^{T} \int_{0}^{t} h(s) d s \int_{\Gamma_{0}} m \cdot \nu|u(t)|^{2} \leq \delta \int_{S}^{T} \int_{\Omega}\left|u_{t}\right|^{2}+C\left(\frac{1}{\delta}+1\right) E(S) \quad \forall 0 \leq S \leq T .
$$

We now estimate the last term of (3.1):

$$
\frac{1}{2} \int_{S}^{T} \int_{\Omega}\left(\left|u_{t}\right|^{2}+|\nabla u|^{2}\right) \leq \delta \int_{S}^{T} \int_{\Omega}\left|u_{t}\right|^{2}+C\left(\frac{1}{\delta}+1\right) E(S) \quad \forall 0 \leq S \leq T .
$$

Choosing $\delta$ sufficiently small, we obtain

$$
\frac{1}{2} \int_{S}^{T} \int_{\Omega}\left(\left|u_{t}\right|^{2}+|\nabla u|^{2}\right) \leq C E(S) \quad \forall 0 \leq S \leq T .
$$

Now from (1.3) and (2.4), we already know that

$$
\int_{S}^{T} h \square u \leq C E(S) \quad \forall 0 \leq S \leq T .
$$

Hence,

$$
\int_{S}^{T} E(t) d t \leq C E(S) \quad \forall 0 \leq S \leq T .
$$

Since $E$ is nonincreasing and satisfies the linear integral inequality (3.7), applying a well-known result of [11] we deduce that $E$ decays exponentially at infinity. This concludes the proof for strong solutions. The exponential decay of weak solutions is obtained by density arguments.

4. Conclusion and open questions. We establish exponential decay of the energy of the solutions of a wave equation subjected to combined frictional and memory boundary dampings. The results presented here concern the exponentially decaying kernel case, but they can be extended to the polynomial case, following partly the techniques introduced in [4], together with the multipliers used in the present paper, in particular the one to estimate the trace of the $L^{2}$ norm of the solution on the part of the boundary where the feedback is active.

Many open questions are of interest. If one considers both nonlinear frictional and polynomially decaying kernels competing, which one will finally determine the optimal decay rate of the energy? How to establish decay rates of the energy for other types of decay rate for the kernel, different than polynomial or exponential? These questions are of interest for boundary as well as internal dampings. Also, if in the wave equation considered in this paper, one removes the frictional damping, it is not clear how to show 
decay of the energy, since we have no further control through dissipation on the kinetic energy of solutions of the part of the boundary on which the feedback is active. A first answer in this direction is given in [5], in which only memory damping is considered. The model for the damping is different from the present one and the convolution kernel is assumed to belong to a class of singular kernels. Such classes of singular kernels have been first introduced in [20]. Uniform stabilization is not expected for pure boundary memory damping. We prove in [5] a polynomial decay rate of the energy.

\section{References}

[1] M. Aassila, M. M. Cavalcanti and V. N. Cavalcanti, Existence and uniform decay of the wave equation with nonlinear boundary damping and boundary memory source term, Calc. Var. Partial Differential Equations 15 (2002), 155-180.

[2] F. Alabau-Boussouira, Convexity and weighted integral inequalities for energy decay rates of nonlinear dissipative hyperbolic systems, Appl. Math. Optim. 51 (2005), 61-105.

[3] - Une formule générale pour le taux de décroissance des systèmes dissipatifs non linéaires, C. R. Math. Acad. Sci. Paris 338 (2004), 35-40.

[4] F. Alabau-Boussouira, P. Cannarsa and D. Sforza, Decay estimates for second order evolution equations with memory, J. Funct. Anal. 254 (2008), 1342-1372.

[5] F. Alabau-Boussouira, J. Prüss and R. Zacher, Exponential and polynomial stability of a wave equation for boundary memory damping with singular kernels, submitted.

[6] P. Cannarsa and D. Sforza, An existence result for semilinear equations in viscoelasticity: the case of regular kernels, in: M. Fabrizio et al. (eds.), Mathematical Models and Methods for Smart Materials, Ser. Adv. Math. Appl. Sci. 62, World Sci., 2002, 343-354.

[7] - - - Semilinear integrodifferential equations of hyperbolic type: existence in the large, Mediterr. J. Math. 1 (2004), 151-174.

[8] M. M. Cavalcanti and H. P. Oquendo, Frictional versus viscoelastic damping in a semilinear wave equation, SIAM J. Control Optim. 42 (2003), 1310-1324.

[9] C. M. Dafermos, Asymptotic stability in viscoelasticity, Arch. Ration. Mech. Anal. 37 (1970), 297-308.

[10] M. Fabrizio and B. Lazzari, On the existence and the asymptotic stability of solutions for linearly viscoelastic solids, ibid. 116 (1991), 139-152.

[11] V. Komornik, Exact Controllability and Stabilization. The Multiplier Method, Masson, Paris, and Wiley, Chichester, 1994.

[12] V. Komornik and E. Zuazua, A direct method for the boundary stabilization of the wave equation, J. Math. Pures Appl. 69 (1990), 33-54.

[13] I. Lasiecka and D. Tataru, Uniform boundary stabilization of semilinear wave equations with nonlinear boundary damping, Differential Integral Equations 8 (1993), $507-533$.

[14] I. Lasiecka and R. Triggiani, Uniform stabilization of the wave equation with Dirichlet or Neumann feedback control without geometrical conditions, Appl. Math. Optim. 25 (1992), 189-224.

[15] P. Martinez, A new method to obtain decay rate estimates for dissipative systems with localized damping, Rev. Mat. Complut. 12 (1999), 251-283. 
[16] J. E. Muñoz Rivera and A. Peres Salvatierra, Asymptotic behaviour of the energy in partially viscoelastic materials, Quart. Appl. Math. 59 (2001), 557-578.

[17] J. E. Muñoz Rivera and M. G. Naso, Asymptotic stability of semigroups associated with linear weak dissipative systems with memory, J. Math. Anal. Appl. 326 (2007), 691-707.

[18] G. Propst and J. Prüss, On wave equation with boundary dissipation of memory type, J. Integral Equations Appl. 8 (1999), 99-123.

[19] J. Prüss, Evolutionary Integral Equations and Applications, Monogr. Math. 87, Birkhäuser, Basel, 1993.

[20] V. Vergara and R. Zacher, Lyapunov functions and convergence to steady state for differential equations of fractional order, Math. Z. 259 (2008), 287-309.

Département de Mathématiques

Université de Metz

INRIA Projet CORIDA

Ile du Saulcy

57045 Metz Cedex 01, France

E-mail: alabau@univ-metz.fr

Received on 13.12.2007;

revised version on 1.4.2008 